\title{
A HOMOLOGICAL PROOF OF A THEOREM BY DAVIS, GERAMITA, ORECCHIA GIVING THE CAYLEY-BACHARACH THEOREM
}

\author{
RICKARD SJÖGREN
}

Let $A$ be a graded Gorenstein $k$-algebra. There is a connection between two Hilbert series related characters for a Cohen-Macaulay (C-M) ideal and its algebraic link in $A$. This was shown by Davis, Geramita, Orecchia [2]. A corollary is the classical Cayley-Bacharach theorem. We will give a homological proof of the D-G-O theorem, using ideas from Peskine-Szpiro [6].

Throughout this paper, let $R=k\left[X_{1}, \ldots, X_{n}\right]$ ( $k$ a field). We call an ideal Gorenstein (C-M) if $R / I$ is Gorenstein (C-M).

We begin by recalling some facts about algebraicaly linked ideals (from e.g. $[6])$.

Definition. Let $G \subset R$ be a Gorenstein ideal, and $I, J \subset R$ ideals with $G \subset I \cap J$. We call $I$ and $J$ algebraically linked by $G$ if

$$
I=G: J \text { and } J=G: I .
$$

i) If this is the case, then ht $I=$ ht $J=h t G$.

ii) Suppose $G=I \cap J$, where $I, J$ are parts of the primary decomposition of $G$ with no common associated prime. Then $I$ and $J$ are algebraically linked.

Next, we recall the definition of the cone of a morphism of complexes, and a long exact sequence involving it (from e.g. [1])

Let $u:\left(C^{\prime}, d^{\prime}\right) \rightarrow(C, d)$ be a morphism of complexes.

Definition. $(\operatorname{Con}(u), D)$ is the complex $(\operatorname{Con}(u))_{n}=C_{n-1}^{\prime} \oplus C_{n}$ and

$$
\begin{aligned}
D: C_{n}^{\prime} \oplus C_{n+1}^{\prime} & \rightarrow C_{n-1}^{\prime} \oplus C_{n} \\
\left(y^{\prime}, x\right) & \mapsto\left(-d^{\prime} y^{\prime}, d x-u y^{\prime}\right)
\end{aligned}
$$

There is a long exact sequence

$$
\ldots \rightarrow H_{n}\left(C^{\prime}\right) \rightarrow H_{n}(C) \rightarrow H_{n}(\operatorname{Con}(u)) \rightarrow H_{n-1}\left(C^{\prime}\right) \rightarrow \ldots
$$


It will prove helpful to establish some additional facts (which can be found in [3], [4]).

Let $A$ be a graded $k$-algebra (i.e. $A=R / J$, for some homogeneous ideal $J$ ) with Krull-dimension $d$ and projective dimension $c$.

1) $A$ has Hilbert-series $H_{A}(z)=p(z) /(1-z)^{d}$.

DEFINITION. $\sigma(A)=\operatorname{deg} p(z)$.

If now $I \subset A$ is a homogeneous ideal, we define the following two characters

Definition. $\sigma(I)=\sigma(A / I)$.

DEFINITION. $\alpha(I)=\min \left\{t \mid I_{t} \neq 0\right\}$.

2) $A$ has a graded $R$-resolution

$$
0 \rightarrow \bigoplus_{i=1}^{b_{c}} R\left[-d_{i, c}\right] \rightarrow \ldots \rightarrow \bigoplus_{i=1}^{b_{1}} R\left[-d_{i, 1}\right] \rightarrow R \rightarrow A \rightarrow 0
$$

where all maps are of degree zero $\left(R[d]_{n}=R_{n+d}\right)$ and are given by forms of positive degree on the components where they are non-zero (it's called a minimal resolution). Conventionally,

$$
d_{1, j} \leqq d_{2, j} \leqq \ldots \leqq d_{b_{j}, j}, \quad \text { all } j .
$$

3) $H_{A}(z)=\left(1-\sum_{1}^{b_{1}} z^{d_{i, 1}}+\ldots+(-1)^{c} \sum_{1}^{b_{c}} z^{d_{i, c}}\right) /(1-z)^{n}$.

4) The sequence of lowest degrees is strictly increasing:

$$
d_{1,1}<d_{1,2}<\ldots<d_{1, c} .
$$

5) If $A$ is $\mathbf{C - M}$, the same for highest degrees:

$$
d_{b_{1}, 1}<d_{b_{2}, 2}<\ldots<d_{b_{c}, c} .
$$

6) If $A$ is Gorenstein, then $b_{c}=1$, and furthermore, the resolution is symmetric, in the sense that $b_{j}=b_{c-j}$, for all $\mathrm{j}$, and if $p(z)=1-\sum z^{d_{i, 1}}+\ldots+(-1)^{c} z^{d_{1, c}}$ then $p(z)=z^{d_{1, c}} p(1 / z)(-1)^{c}$.

7) Here $I$ is any ideal in any noetherian ring $R$.

a) $\operatorname{gr}(I)=$ length of a maximal $R$-sequence in $I=\min \left\{i \mid \operatorname{Ext}_{R}^{i}(R / I, R) \neq 0\right\}$.

b) $\operatorname{gr}(I) \leqq \mathrm{pd}_{R}(R / I)$

c) $R / I \mathrm{C}-\mathrm{M} \Leftrightarrow \operatorname{gr}(I)=\operatorname{pd}_{R}(R / I)$.

We are now appropriately equipped to prove the following

THEOREM. Let $G \subset I$ be homogeneous ideals, $G$ Gorenstein and I C-M with the same dimension in $R=k\left[X_{1}, \ldots, X_{n}\right]$. Let $J=G: I$. Then

$$
\alpha(\bar{J})+\sigma(\bar{I})=\sigma(\bar{R}) \quad(\bar{X}=X / G) .
$$


REMARK. Then, also, $\alpha(\bar{I})+\sigma(\bar{J})=\sigma(\bar{R})$ since also I satisfies the hypothesis and $I, J$ are algebraically linked by $G$, according to [6].

Proof. Put $\operatorname{dim}(R / I)=\operatorname{dim}(R / G)=n-k$ (so both $I$ and $G$ have height $k$ ). Then $\operatorname{pd}(R / G)=\operatorname{pd}(R / I)=k$ (use (7)). Take minimal graded $R$-resolutions $\mathscr{F}_{0}$, $\mathscr{F}_{1}$ of $R / G$ and $R / I$, respectively.

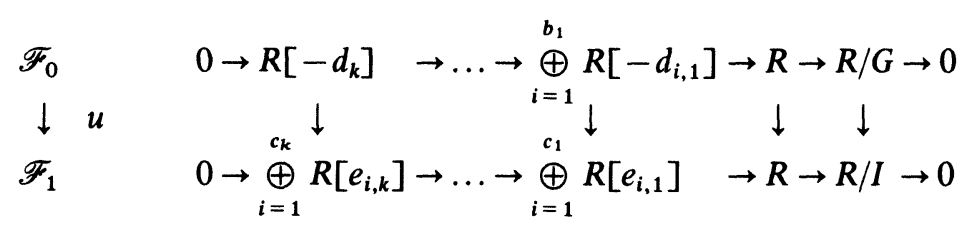

The lifting $u$ exists (well-known).

Note. The resolutions enable us to deduce the Hilbert-series (3), e.g. $H_{R / G}(z)=p(z) /(1-z)^{n}$ with $p(z)$ as in (6). This allows us to compute the $\sigma$ 's; $\sigma(R / G)=d_{k}-k, \sigma(I)=e_{c_{k}, k}-k$.

Dualize, that is, apply the functor $\operatorname{Hom}_{R}(., R)$ (remember $\operatorname{Hom}(R[-a], R)=R[a])$.

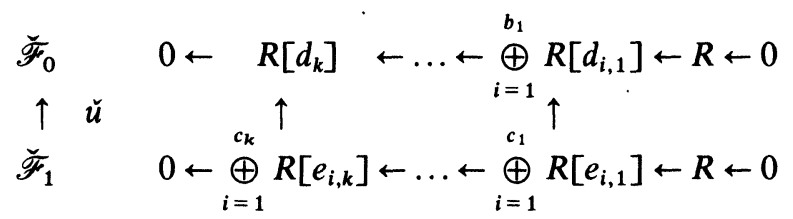

Here, let the homological dimension decrease from $k$ (to the right) to 0 (left). Now, take the cone.

$\operatorname{Con}(\check{u}) 0 \leftarrow R\left[d_{k}\right] \leftarrow \bigoplus_{i=1}^{c_{k}} R\left[e_{i, k}\right] \bigoplus_{i=1}^{b_{k}-1} R\left[d_{i, k-1}\right] \leftarrow \ldots \leftarrow \bigoplus_{i=1}^{c_{1}} R\left[e_{i, 1}\right] \oplus R \leftarrow R \leftarrow 0$

Shift degrees.

$$
\begin{aligned}
\operatorname{Con}(\breve{u})\left[-d_{k}\right] & 0 \leftarrow R \leftarrow \bigoplus_{i=1}^{c_{k}} R\left[e_{i, k}-d_{k}\right] \bigoplus_{i=1}^{b_{k}-1} R\left[d_{i, k-1}-d_{k}\right] \leftarrow \ldots \\
& \leftarrow \bigoplus_{i=1}^{c_{1}} R\left[e_{i, 1}-d_{k}\right] \oplus R\left[-d_{k}\right] \leftarrow R\left[-d_{k}\right] \leftarrow 0 .
\end{aligned}
$$

CLAIM. $\operatorname{Con}(\check{u})\left[-d_{k}\right] \rightarrow R / J \rightarrow 0$ is a resolution.

Before proving the claim, let's wrap up.

$$
\begin{aligned}
(1-z)^{n} H_{R / J}(z)= & \\
= & 1-\sum z^{d_{k}-d_{i, k-1}}+\ldots+(-1)^{k-1} \sum z^{d_{k}-d_{i, 1}}+(-1)^{k} z^{d_{k}} \\
& -\sum z^{d_{k}-e_{i, k}}+\ldots+(-1)^{k} \sum z^{d_{k}-e_{i, 1}}+(-1)^{k+1} z^{d_{k}}
\end{aligned}
$$


The first row on the right side is $z^{d_{k}} p(1 / z)(-1)^{k}=p(z)$, so

$$
H_{R / J}(z)=H_{R / G}(z)-\frac{\sum z^{d_{k}-e_{i, k}}-\ldots+(-1)^{k-1} \sum z^{d_{k}-e_{i, 1}}+(-1)^{k} z_{k}^{d}}{(1-z)^{n}} .
$$

Consider this exact sequence of graded $R$-modules:

$$
0 \rightarrow J / G \rightarrow R / G \rightarrow R / J \rightarrow 0 .
$$

It follows that

$$
H_{J / G}(z)=H_{R / G}(z)-H_{R / J}(z)=\frac{\sum z^{d_{k}-e_{i, k}}-\ldots+(-1)^{k-1} \sum z^{d_{k}-e_{i, 1}}+(-1)^{k} z_{k}^{d}}{(1-z)^{n}} .
$$

Now,

$$
\begin{aligned}
\alpha(J / G) & =\text { the least occuring degree in the numerator }= \\
& =d_{k}-e_{c_{k}, k} \quad(R / I \mathrm{C}-\mathrm{M}, \text { use (5)). }
\end{aligned}
$$

But, as pointed out in the note above,

$$
\left\{\begin{array}{l}
\sigma(\bar{R})=d_{k}-k \\
\sigma(\bar{I})=\sigma(I)=e_{c_{k}, k}-k
\end{array}\right.
$$

so

$$
\alpha(J / G)=\sigma(\bar{R})-\sigma(\bar{I}) .
$$

The proof is completed, but for the proof of the claim. Before proceeding with that, we recall from [5, Lemma 2], that there is a functorial isomorphism

$$
\operatorname{Ext}_{R}^{k}(M, R) \simeq \operatorname{Hom}_{R}(M, R / G)
$$

for modules $M$ with $\operatorname{Ann}(M) \supset G$.

Proof of Claim. We have to show

$$
\left\{\begin{array}{l}
H_{0}(\text { Con })=R / J \\
H_{i}(\text { Con })=0, \quad i>0
\end{array}\right.
$$

Use the characterization of grade by Ext (7a) to get

$$
\left\{\begin{array} { l l } 
{ \operatorname { E x t } _ { R } ^ { i } ( R / G , R ) = 0 , } & { i < k } \\
{ \operatorname { E x t } _ { R } ^ { i } ( R / I , R ) = 0 , } & { i < k }
\end{array} \text { , whence } \left\{\begin{array}{ll}
H_{i}\left(\check{\mathscr{F}}_{0}\right)=0, & i>0 \\
H_{i}\left(\check{\mathscr{F}}_{1}\right)=0, & i>0
\end{array}\right.\right.
$$

Consider the long exact sequence mentioned in context with the definition of the cone

$$
H_{i}\left(\check{\mathscr{F}}_{0}\right) \rightarrow H_{i}(\text { Con }) \rightarrow H_{i-1}\left(\mathscr{F}_{1}\right) .
$$


From this, we immediately get

$$
i>1 \Rightarrow H_{i}(\text { Con })=0 .
$$

We also have, further down in the exact sequence,

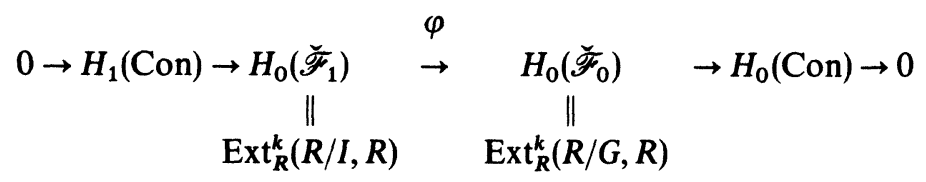

But there is a commutative diagram

$$
\begin{aligned}
& 1_{\mathrm{xt}}^{k}(R / I, R) \simeq \operatorname{Hom}_{R}(R / I, R / G)=\operatorname{Hom}_{R / G}(R / I, R / G) \\
& \downarrow \varphi \quad \downarrow \operatorname{Hom}_{R / G}\left(\frac{R / G}{I / G}, R / G\right) \simeq \frac{G: I}{G} \\
& \operatorname{Ext}_{R}^{k}(R / G, R) \simeq \operatorname{Hom}_{R}(R / G, R / G)=\operatorname{Hom}_{R / G}(R / G, R / G) \simeq \quad
\end{aligned}
$$

Let's take a moment to explain this. One gets the first quadrant by applying (*) above. The equalities are true, since $G \subset \operatorname{Ann}(R / I) \cap \operatorname{Ann}(R / G)$, and the last isomorphism in the top row is due to the fact that $\operatorname{Hom}_{S}(S / J, S) \simeq 0: J$ for any ring $S$. Furthermore, $\beta$ is mono, since the functor $\operatorname{Hom}(\cdot R / G)$ is left exact. Now, we have

$$
\begin{aligned}
& \beta \text { mono } \Rightarrow \varphi \text { mono } \Rightarrow H_{1}(\text { Con })=0 \\
& H_{0}(\text { Con })=\operatorname{Coker}\left(\frac{G: I}{G} \rightarrow R / G\right)=\frac{R}{G: I}=R / J .
\end{aligned}
$$

This ends proof of claim.

A rather immediate consequence of this theorem is the

Cayley-Bacharach theorem. Let $\left(f_{1}, \ldots, f_{r}\right)$ be a complete intersection in $k\left[X_{0}, X_{1}, \ldots, X_{r}\right]\left(k\right.$ alg. closed field), $\operatorname{deg} f_{i}=d_{i}$ and $V\left(f_{1}, \ldots, f_{r}\right)=$ $\left\{P \in \mathscr{P r} \mid f_{i}(P)=0 \forall i\right\}=\left\{P_{1}, \ldots, P_{m}\right\}$. Suppose $m=d_{1} \cdot \ldots \cdot d_{r}$. Then every hypersurface of degree $\leqq \sum d_{i}-\gamma(\gamma \geqq r+1)$ which passes through $m-\left(\begin{array}{c}\gamma-1 \\ r\end{array}\right)$ of the points in $V$, passes through them all, if the remaining $\left(\begin{array}{c}\gamma-1 \\ r\end{array}\right)$ don't lie on a hypersurface of degree $\leqq \gamma-r-1$.

This is proved in [2]. Of course, the first proof, for $r=2$, was done in the 1880's, with completely different methods. 


\section{REFERENCES}

1. N. Bourbaki, Algèbre, chap. 10, (Algébre homologique) Masson 1982.

2. E. D. Davis, A. V. Geramita, F. Orecchia, Hilbert functions of linked varieties, Queens paper in pure and applied math. no 67, 1984.

3. R. Fröberg and D. Laksov, Compressed algebras, SLN 1092, 121-151 1983.

4. A. Geramita and C. Small, Introduction to homological methods in commutative rings, Queens paper in pure and applied math. no 43, 1976.

5. E. S. Golod, A note on perfect ideals, Algebra (A. I. Kostrikin ed.), Moscow State Univ. Publishing House, 37-39 1980.

6. C. Peskine et L. Szpiro, Liaison des variétes algébriques I, Invent. Math. 26 1974, 271-302.

7. R. Stanley, Hilbert functons of graded algebras, Adv. in Math. 28 1978, 57-83.

\section{DEPARTMENT OF MATHEMATICS}

UIVERSITY OF STOCKHOLM

BOX 6701 .

11385 STOCKHOLM

SWEDEN 